\title{
Optimal activation of tumor-reactive $T$ cells by selected antigenic peptide analogues
}

\author{
Danila Valmori, Jean-Francois Fonteneau', Salvatore Valitutti ${ }^{2}$, Nadine Gervois ${ }^{1}$, \\ Rod Dunbar ${ }^{3}$, Danielle Liénard, Donata Rimoldi, Vincenzo Cerundolo ${ }^{3}$, Francine \\ Jotereau', Jean-Charles Cerottini, Daniel E. Speiser and Pedro Romero \\ Division of Clinical Onco-immunology, Ludwig Institute for Cancer Research, Lausanne Branch, University \\ of Lausanne, CHUV, 1011 Lausanne, Switzerland \\ 1 Unit 463, Institut National de la Santé et de la Recherche Médicale, F-44035 Nantes, France \\ ${ }^{2}$ Institute of Biochemistry, University of Lausanne, 1066 Epalinges, Switzerland \\ ${ }^{3}$ Institute of Molecular Medicine, Nuffield Department of Medicine, John Radcliffe Hospital, Oxford OX3 \\ 9DU, UK
}

Keywords: cytotoxicity, cytotoxic T lymphocyte, T lymphocytes, tumor immunity, vaccination

\begin{abstract}
Many mechanisms have been proposed to explain why immune responses against human tumor antigens are generally ineffective. For example, tumor cells have been shown to develop active immune evasion mechanisms. Another possibility is that tumor antigens are unable to optimally stimulate tumor-specific T cells. In this study we have used HLA-A2/Melan-A peptide tetramers to directly isolate antigen-specific $\mathrm{CD8}^{+} \mathrm{T}$ cells from tumor-infiltrated lymph nodes. This allowed us to quantify the activation requirements of a representative polyclonal yet monospecific tumorreactive $T$ cell population. The results obtained from quantitative assays of intracellular $\mathrm{Ca}^{2+}$ mobilization, TCR down-regulation, cytokine production and induction of effector cell differentiation indicate that the naturally produced Melan-A peptides are weak agonists and are clearly suboptimal for $T$ cell activation. In contrast, optimal $T$ cell activation was obtained by stimulation with recently defined peptide analogues. These findings provide a molecular basis for the low immunogenicity of tumor cells and suggest that patient immunization with full agonist peptide analogues may be essential for stimulation and maintenance of anti-tumor $\mathrm{T}$ cell responses in vivo.
\end{abstract}

\section{Introduction}

Tumor-reactive CD8 ${ }^{+}$cytotoxic T lymphocytes (CTL) derived from tumor-infiltrating lymphocytes (TIL) of melanoma patients often recognize unmodified self peptides derived from melanocyte lineage-specific antigens bound to MHC class I molecules $(1,2)$. In particular, significant CTL responses specific for an immunodominant epitope derived from the Melan-A/MART1 protein can be frequently measured in HLA-A*0201 melanoma patients (3-8). Although significant levels of specific tumoricidal CTL expressing an antigen-experienced phenotype can be detected ex vivo in TIL from melanoma patients (9), the functional status of these cells in vivo and their impact on tumor growth remain unclear since many patients develop progressive disease despite the presence of infiltrating tumoricidal CTL.

In order to explain the apparent paradox between detection of anti-tumor CTL responses and tumor progression, different mechanisms of tumor immune evasion have been described (10-14). Moreover, it has been proposed that tumor-reactive CTL could be stimulated only suboptimally by relevant tumor antigens. In vitro, co-culture of melanoma cells and TIL generally results in lysis of the antigen-bearing tumor cells but inefficient proliferation and $\mathrm{IL}-2$ secretion by responder $\mathrm{T}$ cells (15). This suboptimal activation has been classically explained by the inability of tumor cells to provide costimulatory signals. More recent results suggest that expression of MHC-peptide complexes at a subthreshold level could also at least partially explain this phenomenon (16). The characteristics of the peptide ligand itself have been shown to greatly influence the nature and magnitude of $T$ cell responses by providing suboptimal or optimal stimulation

The first two authors contributed equally to this work and the last two authors share senior authorship

Correspondence to: D. Valmori

Transmitting editor: P. C. L. Beverley

Received 13 July 1999, accepted 24 August 1999 
(17-20). Many studies have been performed to improve our understanding of the interaction between TCR, peptide and $\mathrm{MHC}$ antigens, as well as the consequences on cellular activation and effector functions such as $T$ cell proliferation, cytokine production and induction of effector cells $(21,22)$. However, such studies were performed with monoclonal $T$ cell populations or TCR transgenic animals which are only partially representative of the whole $T$ cell repertoire involved in an immune response. The possibility to perform these analyses on normal polyclonal antigen populations has been thus far hampered by the difficulty of directly isolating antigenspecific T cells.

We have recently produced fluorescent A2/Melan-A peptide tetramers to directly visualize and isolate Melan-A-specific $T$ cells from tumor-infiltrated lymph nodes (TILN) and peripheral blood mononuclear cells (PBMC) of HLA-A*0201 melanoma patients (9). In the present study we investigated the capacity of synthetic peptides corresponding to the Melan-A immunodominant HLA-A2-restricted CTL epitope as well as their recently described enhanced analogues (23-25) to activate a polyclonal monospecific T cell population derived from TILN by sorting according to tetramer staining. We found that the natural Melan-A peptides, although they were able to efficiently sensitize target cells for lysis, were suboptimal at inducing intracellular $\mathrm{Ca}^{2+}$ mobilization, TCR down-regulation and cytokine expression by antigen-specific T cells. In contrast, the peptide analogues were able to induce rapid, strong and full $\mathrm{T}$ cell activation. These results indicate that the parental Melan-A peptides, although immunodominant, are only weak agonists, and thus suboptimal for stimulation and maintenance of anti-tumor $\mathrm{T}$ cell responses.

\section{Methods}

\section{Peptides}

The following synthetic Melan-A peptides were used: the parental nonamer AAGIGILTV (Melan- $A_{27-35}$ ), the parental decamer EAAGIGILTV (Melan-A $26-35)$, and the decamer analogues ELAGIGILTV, AAAGIGILTV and ALAGIGILTV. All peptides were synthesized by standard solid-phase chemistry on a multiple peptide synthesized (Applied Biosystems, Foster City, CA) by using F-moc for transient $\mathrm{N}$-terminal protection and were analyzed by mass spectrometry. All peptides were $>90 \%$ pure as indicated by analytical HPLC. Lyophilized peptides were diluted in DMSO and stored at $-20^{\circ} \mathrm{C}$.

\section{Cells and cultures}

The human mutant cell line CEMx721.174.T2 (T2) used as target and presenting cells (26) was cultured in RPMI containing 10\% FCS. Lymphocyte cultures were generated from samples obtained from the melanoma patient LAU 203 who was HLA-A*0201. TILN were generated from tumor-invaded lymph nodes obtained by surgery at the Centre Pluridisciplinaire d'Oncologie, CHUV, Lausanne, Switzerland. TILN fragments were minced to single-cell suspensions and cultured in 24-well tissue plates (Costar, Cambridge, MA) in $2 \mathrm{ml}$ of IMDM supplemented with Asn, Arg and GIn, and 10\% pooled human $A^{+}$serum in the presence of rIL-2 and rIL-7 (100 U/ $\mathrm{ml}$ and $10 \mathrm{ng} / \mathrm{ml}$ respectively). After 16 days in culture, their surface phenotype was determined by flow cytometry. $\mathrm{CD}^{+}{ }^{+} \mathrm{CD}^{+}{ }^{+}$TILN were sterile sorted into A2/Melan-A peptide tetramer-positive and -negative populations. After 2 weeks of culture in the presence of irradiated allogeneic PMBC and phytohemaglutinin, the tetramer-positive population had expanded considerably. It was cryopreserved in multiple aliquots, thus providing a stock of tumor antigen-specific lymphocytes that allowed us to perform and reproduce multiple assays. After thawing, the cells were cultured for an additional 2-3 days prior to use in the different functional assays. The CTL clone 1.13 was derived from PBMC of a normal HLA-A2 donor after in vitro re-stimulation with peptide Melan- $A_{27-35}$ as previously described (24). Stimulation of blood-derived lymphocytes was performed as follows. PBMC were isolated by centrifugation on Ficoll-Paque (Pharmacia, Uppsala, Sweden) and plated at $10 \times 10^{6}$ cells/well in $2 \mathrm{ml}$ of IMDM as for the TILN (see above). The PBMC were then stimulated weekly with autologous PBMC $\left(3 \times 10^{6} /\right.$ well $)$ pulsed during $2 \mathrm{~h}$ at $37^{\circ} \mathrm{C}$ in serum-free medium (X-VIVO 10; BioWhittaker, Walkersville, MD) with the appropriate peptide $(1 \mu \mathrm{M})$ and human $\beta_{2}$-microglobulin $(3 \mu \mathrm{g} / \mathrm{ml})$. Peptide-pulsed PBMC were then washed 2 times, irradiated (3000 rad) and adjusted to the appropriate volume before addition to the responder cell population. IL-2 (10 U/ml) and IL-7 (10 ng/ml) were added during the first two stimulation cycles, and IL-2 alone (100 U/ $\mathrm{ml}$ ) during the third week.

\section{Tetramers}

Complexes were synthesized as described $(9,27)$. Briefly, purified HLA heavy chain and $\beta_{2}$-microglobulin were synthesized by means of a prokaryotic expression system (pET; R \&D Systems, Minneapolis, MN). The heavy chain was modified by deletion of the transmembrane-cytosolic tail and C-terminal addition of a sequence containing the BirA enzymatic biotinylation site. Heavy chain, $\beta_{2}$-microglobulin and peptide were refolded by dilution. The $45 \mathrm{kDa}$ refolded product was isolated by fast protein liquid chromatography and then biotinylated by BirA (Avidity, Denver, CO) in the presence of biotin (Sigma, St Louis, MO), adenosine $5^{\prime}$-triphosphate (Sigma) and $\mathrm{Mg}^{2+}$ (Sigma). Streptavidin-phycoerythrin conjugate (Sigma) was added in a 1:4 molar ratio and the tetrameric product was concentrated to $1 \mathrm{mg} / \mathrm{ml}$. The Melan- $\mathrm{A}_{26-35} \mathrm{~A} 27 \mathrm{~L}$ analogue (ELAGIGILTV) was used as antigenic peptide because it has a higher binding stability to HLA-A*0201, and a higher $T$ cell antigenicity and immunogenicity than the natural Melan-A decapeptide EAAGIGILTV or the nonapeptide AAGIGILTV (23). The interchangeability of tetramers synthesized around the Melan-A A27L modified peptide and around the parental Melan-A peptide for staining of Melan-A-specific T cells has been previously assessed (9). In this study, the abbreviation A2/Melan-A peptide tetramers is used for the HLA-A*0201/ Melan- $A_{26-35}$ A27L tetramers.

\section{Cytolytic activity assay}

Standard $4 \mathrm{~h}{ }^{51} \mathrm{Cr}$-release assays were used to assess antigen-specific target cell lysis. Target cells were labeled with sodium $\left[{ }^{51} \mathrm{Cr}\right]$ chromate in Tris-Dulbecco buffer supplemented with $2 \mathrm{mg} / \mathrm{ml}$ BSA for $1 \mathrm{~h}$ at $37^{\circ} \mathrm{C}$ and washed twice. Labeled target cells ( 1000 cells in $50 \mu \mathrm{l}$ ) were incubated in the presence of various concentrations of peptide $(50 \mu \mathrm{l})$ for 15 
min at room temperature before the addition of CTL at a lymphocyte:target cell ratio of 10:1. Chromium release was measured after incubation for $4 \mathrm{~h}$ at $37^{\circ} \mathrm{C}$. The percent specific Iysis was calculated as: $100 \times$ (experimental - spontaneous release)/(total - spontaneous release).

\section{Calcium mobilization}

To measure intracellular calcium $\left[\mathrm{Ca}^{2+}\right]$ cells were loaded with Indo-1 AM (Sigma) as described (28). T cells were mixed at a 1:2 ratio with T2 cells that had been pulsed for $2 \mathrm{~h}$ at $37^{\circ} \mathrm{C}$ with serial dilutions of the different peptides. The cells were centrifuged ( $1 \mathrm{~min}$ at 1500 r.p.m.), incubated $1 \mathrm{~min}$ at $37^{\circ} \mathrm{C}$, resuspended and analyzed by flow on a FACStar cytofluorometer (Becton Dickinson, Mountain View, CA) by gating on a forward light scattering corresponding to cell conjugates.

$m A b$

Phycoerythrin-conjugated MQ1-17H12 (anti-IL-2), Mab11 [anti-tumor necrosis factor (TNF)- $\alpha$ ], BVD2-23B6 (anti-GMCSF), MP4-25D2 (anti-IL-4), 4S.B3 (anti-IFN- $\gamma$ ) and FITCconjugated 4S.B3 (anti-IFN- $\gamma$ ) purified antibodies were obtained from PharMingen (San Diego, CA). Phycoerythrinconjugated OKT3 (anti-CD3) purified antibody was obtained from Immunotech (Marseille, France).

Staining of CD3 and intracellular cytokines by flow cytometry For CD3 down-regulation experiments, T2 cells were pulsed for $1 \mathrm{~h}$ at $37^{\circ} \mathrm{C}$ with different peptide concentrations and washed twice to avoid autopresentation by $T$ cells. $T$ cells $\left(1 \times 10^{5}\right)$ were stimulated by peptide-pulsed T2 cells $\left(2 \times 10^{5}\right)$. After $6 \mathrm{~h}$ incubation at $37^{\circ} \mathrm{C}$, cells were stained for CD3, acquired on a FACScan cytofluorometer (Becton Dickinson) and analyzed using Lysys II software (Becton Dickinson). Logarithmically amplified fluorescence data were collected for intact lymphocytes as determined by scatter gates. Percentage of CD3 down-regulation was determined as: (nonstimulated T cell CD3 mean of fluorescence - stimulated T lymphocyte CD3 mean of fluorescence/non-stimulated T cell CD3 mean of fluorescence) $\times 100$. For intracellular staining of cytokines and co-staining of CD3 and intracellular cytokines, $\mathrm{T}$ cell clones or T cell lines $\left(4 \times 10^{5}\right)$ were stimulated $6 \mathrm{~h}$ by coculture with peptide pulsed T2 cells $\left(8 \times 10^{5}\right)$ in the presence of $10 \mu \mathrm{g} / \mathrm{ml}$ of Brefeldin A (Sigma) to inhibit cytokine secretion. For intracellular staining of cytokines, cells were fixed for 10 min at room temperature in a solution of PBS/4\% paraformaldehyde and were stained for intracellular cytokine. Fixed stimulated $T$ cells were stained for cytokines as described (29). Briefly, $5 \times 10^{4} \mathrm{~T}$ cells were stained with the different $\mathrm{mAb}$ at concentration of $5 \mu \mathrm{g} / \mathrm{ml}$ for $30 \mathrm{~min}$ at room temperature. Reagent dilutions and washes were made with PBS containing $0.1 \%$ BSA (Sigma) and 0.1\% saponin (Sigma). After staining, cells were resuspended in PBS and analyzed on a FACScan using CellQuest software (Becton Dickinson).

\section{Results}

Generation of a polyclonal Melan-A monospecific $T$ cell population from TILN of a melanoma patient

In order to obtain tumor antigen-specific T cells, we prepared single-cell suspensions from TILN as previously described
A



B

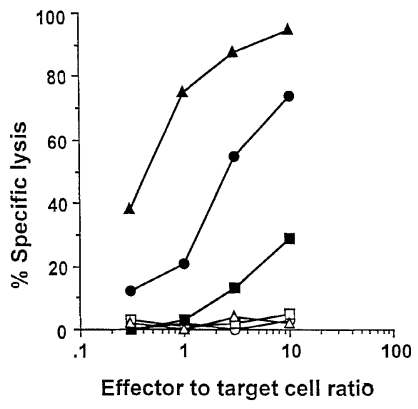

Fig. 1. Generation of a polyclonal Melan-A monospecific $T$ cell population from TILN. (A) The TILN population from patient LAU 203 was analyzed after 16 days in culture with rIL-2 and rIL-7 by flow cytometry using A2/Melan-A tetramers as described (9) together with anti-CD8-FITC and anti-CD3-PerCP. The TILN contained $>90 \%$ $\mathrm{CD}^{+}{ }^{+} \mathrm{CD} 8^{+}$cells and $19.4 \% \mathrm{CD}^{+}$tetramer-positive cells (top panel). $\mathrm{CD}^{+}{ }^{+} \mathrm{CD} 8^{+}$TILN were sterile sorted into A2/Melan-A tetramer-positive and tetramer-negative populations, and expanded during 2 weeks in the presence of irradiated allogeneic PBMC, phytohemagglutinin, and $\mathrm{rlL}-2+\mathrm{rlL}-7$. After this, the tetramer-positive cell fraction contained $94.4 \%$ (middle panel) and the tetramer-negative culture $1.1 \%$ (lower panel) tetramer-positive cells. The dot plots show all small lymphocytes as defined by forward scatter/side scatter gating. (B) The three cell populations, i.e. total TILN LAU 203 (circles), A2/Melan-A tetramer-positive fraction (triangles) and A2/Melan-A tetramer-negative fraction (squares), were tested for lytic activity against ${ }^{51} \mathrm{Cr}$-labeled T2 target cells in absence (open symbols) or presence of peptide Melan-A 26-35 (1 $\mu \mathrm{M})$ (closed symbols) as indicated.

(30) and cultured them for 2 weeks in medium containing rIL2 and $\mathrm{rlL}-7$. In the majority of $\mathrm{HLA}-\mathrm{A}^{*} 0201$-positive patients, this resulted in a strong enrichment of tumor-specific $T$ cells specific for the immunodominant Melan-A peptide (E)AAGIGILTV. In this study, we investigated the TILN cells from patient LAU 203 in detail. After 16 days of culture, the cells were analyzed by three-color flow cytometry using A2/Melan-A peptide-phycoerythrin tetramers (9) together with anti-CD3PerCP and anti-CD8-FITC. The TILN contained $>90 \%$ $\mathrm{CD}^{+}{ }^{+} \mathrm{CD} 8^{+}$cells. A high percentage $(19.4 \%)$ of $\mathrm{CD}^{+}{ }^{+} \mathrm{CD} 8^{+}$ A2/Melan-A tetramer-positive cells were detected in the total TILN population (Fig. 1A, upper panel). Through sterile sorting we obtained $\mathrm{CD}^{+} \mathrm{A} 2 /$ Melan-A peptide tetramer-positive and $\mathrm{CD}^{+}$A2/Melan-A peptide tetramer-negative populations. After expansion during 2 weeks in the presence of irradiated allogeneic PBMC and phytohemagglutinin, the two cultures were stained with A2/Melan-A peptide-phycoerythrin tetramers and anti-CD8-FITC (Fig. 1A, middle and lower panel). Cultures were then tested in a ${ }^{51} \mathrm{Cr}$-release assay. No lysis of T2 target cells was detected in absence of the antigenic peptide. As compared to the total, unfractionated TILN population, the A2/Melan-A peptide tetramer-positive cells showed an enhanced level of antigen-specific lysis that was approximately proportional to the degree of antigen-specific $\mathrm{T}$ cell enrichment. A low level of antigen-specific lysis was also 
detected for the A2/Melan-A peptide tetramer-negative cell fraction, which may be explained by the presence of a small percentage of contaminating A2/Melan-A peptide tetramerpositive $T$ cells in this population (Fig. 1B).

Fine specificity analysis of the Melan-A-specific T cells

Parental Melan-A immunodominant peptides and the recently described Melan-A peptide analogues $(23,24)$ were used to compare the fine specificity of unsorted TILN with that of the sorted A2/Melan-A peptide tetramer-positive cells (Fig. 2A and $\mathrm{B}$ ). Both effector populations induced similar lysis of T2 target cells in the presence of titrated concentrations of MelanA parental and analogue peptides. Half-maximal lysis was obtained with $\sim 1.5 \times 10^{-7} \mathrm{M}$ of parental nonapeptide while the parental decapeptide was recognized 5 -fold better. The Melan-A analogues $(23,24)$ were recognized much more efficiently, since half-maximal lysis was obtained at peptide concentrations as low as $10^{-10}$ to $10^{-11} \mathrm{M}$. These data demonstrate a similar fine specificity in both unsorted and sorted TILN, confirming our earlier findings $(23,24)$. Thus, the A2/Melan-A peptide tetramer-positive sorted cell population can be considered as a polyclonal, yet monospecifc $\mathrm{CD}^{+} \mathrm{T}$ cell population which is representative of the total antigenspecific T cells.

Early activation events: TCR down-regulation and $\mathrm{Ca}^{2+}$ mobilization

The tetramer-positive cells were used for a detailed study of $T$ cell activation and effector function. TCR down-regulation is one of the earliest $T$ cell activation events induced upon antigen recognition and subsequent TCR triggering. As shown in Fig. 2(C), the parental Melan-A peptides induced only weak TCR down-regulation even at the high peptide doses that were efficient in target cell lysis (Fig. 2B). In contrast, maximal TCR down-regulation was induced by the peptide analogues. Nevertheless, TCR down-regulation always required much higher peptide doses than target cell lysis, confirming earlier findings $(21,31)$.

We also measured the $\mathrm{Ca}^{2+}$ response which occurs early after TCR engagement. The Melan-A-specific T cell clone 1.13 (24) was included as a positive control. $\mathrm{Ca}^{2+}$ flux was measured in response to different concentrations of the parental decapeptide and the analogue peptide ELAGIGILTV. As shown in Fig. $3(A)$, the analogue but not the parental peptide triggered a full $\mathrm{Ca}^{2+}$ response. While the clone required a distinct peptide analogue concentration of $\sim 1 \mathrm{ng} /$ $\mathrm{ml}$, the polyclonal tetramer-positive cells were triggered for $\mathrm{Ca}^{2+}$ flux at a broader range of peptide analogue concentrations, with some cells already reacting at the lowest peptide concentration tested $\left(10^{-3} \mathrm{ng} / \mathrm{ml}\right)$. Furthermore, at the maximal peptide concentration, the parental decapeptide triggered $\mathrm{Ca}^{2+}$ flux in only $60 \%$ of the CTL clone 1.13 and $90 \%$ of the tetramer-positive population, whereas the analogue peptide mobilized $\mathrm{Ca}^{2+}$ in nearly $100 \%$ of both cell populations. Moreover, the data show that $\sim 50 \%$ of TCR down-regulation was required to attain a full $\mathrm{Ca}^{2+}$ response. Some typical $\mathrm{Ca}^{2+}$ responses are shown in Fig. 3(B). The fact that $90 \%$ of the tetramer-positive population exhibited increased intracellular $\mathrm{Ca}^{2+}$ in response to the parental decapeptide also confirmed that this population was highly specific for the parental


Fig. 2. Target cell lysis and TCR down-regulation induced by parental versus peptide analogues at various peptide concentrations. The cytolytic activity by unsorted TILN (A) versus tetramer-positive TILN (B) was tested against T2 target cells labeled with titrated peptide concentrations. Both effector populations recognized the parental peptides (circles) less efficiently than each of the three peptide analogues (squares and triangles). The hierarchy of peptide recognition was similar between the two effector populations. Analysis of TCR down-regulation (C) in the tetramer-positive population revealed again a comparable peptide hierarchy. However, as compared to the lysis data (B), TCR down-regulation of tetramerpositive TILN required higher peptide concentrations and only poor down-regulation was achieved by the parental Melan-A peptides (circles).

peptide. Taken together, the activation hierarchy of the peptides was similar for TCR down-regulation, $\mathrm{Ca}^{2+}$ response and cytotoxicity.

Late activation events: cytokine synthesis and induction of cytolytic effector function

Intracellular staining of cytokines is a very sensitive method to determine the cytokine response at the single cell level (29). We used this method to measure the TNF- $\alpha$, IFN- $\gamma$, IL- 


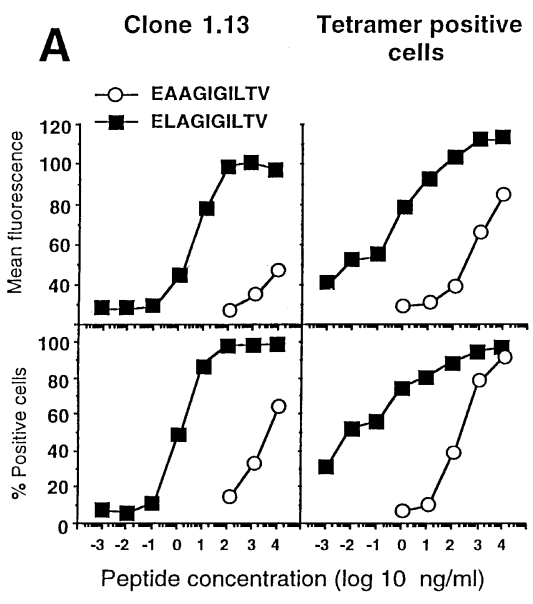

B Clone 1.13 Tetramer positive cells

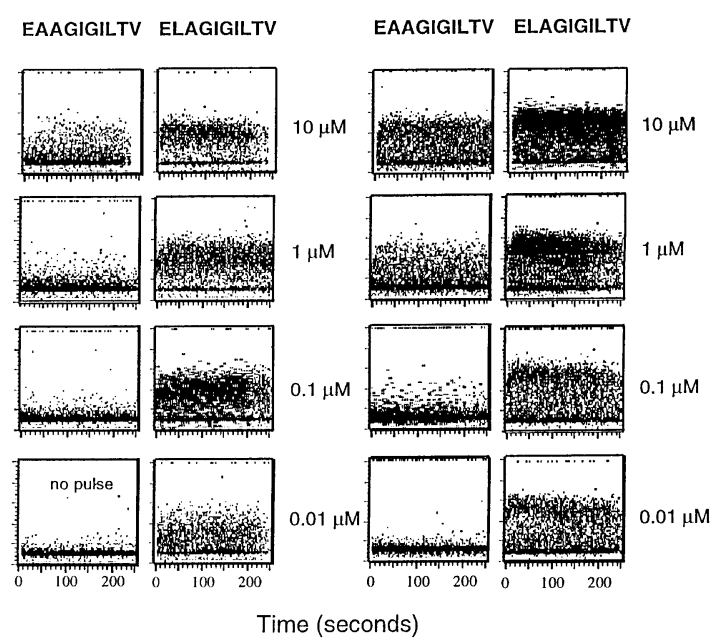

Fig. 3. Induction of intracellular $\mathrm{Ca}^{2+}$ flux by Melan-A parental and analogue peptides. The Melan-A-specific T cell clone 1.13 and the tetramerpositive population were stimulated and analyzed for $\mathrm{Ca}^{2+}$ mobilization as described in Methods $(\mathrm{A})$. As compared to the parental peptide, the analogue peptide ELAGIGILTV induced a higher $\mathrm{Ca}^{2+}$ flux (mean fluorescence) by a higher percentage of cells (B).

2, granulocyte macrophage colony stimulating factor and IL4 production by the CTL clone 1.13 and the tetramer-positive cells in response to different concentrations of parental or analogue peptides. The two parental peptides only stimulated small fractions of the populations tested. For example, only $30 \%$ of the CTL clone 1.13 produced TNF- $\alpha$ at the highest parental peptide concentration. Likewise, 10 and $45 \%$ of the tetramer-positive population produced $\mathrm{TNF}-\alpha$ in response to the highest concentrations of parental nonapeptide and decapeptide respectively. In contrast, the analogue peptide stimulated a much larger fraction of CTL able to produce cytokines. A plateau of response was reached at $1 \mu \mathrm{M}$ for the two populations (Fig. 4A). However, none of the peptides stimulated the entire clonal or polyclonal CTL populations (Fig. 4A), consistent with our previous observation that a fraction of CTL was refractory to cytokine production (32). We always observed a lower percentage of IL-2 and IL-4 producing cells than TNF- $\alpha$-producing cells at all tested peptide concentrations. The tetramer-positive population was distinguishable from the CTL clone 1.13 by a greater ability to produce IFN- $\gamma$ and an incapacity to produce IL-4. This is consistent with our earlier observations of great interclonal heterogeneity in cytokine production by different T cells (32). To better visualize heterogeneity of the population analyzed in cytokine production and in the quantity of cytokines produced per cell, we performed IFN- $\gamma /$ TNF- $\alpha$ and IFN- $\gamma /$ IL-2 double stainings on the two populations after stimulation with $10 \mu \mathrm{M}$ of each of the three peptides (Fig. 4B). The parental peptides stimulated low fractions of $T$ cells to produce TNF$\alpha, \mathrm{IFN}-\gamma$ and IL-2. When the cells were stimulated with the analogue peptides both the percentages of cells producing cytokines and the amount of cytokines per cell were greatly enhanced as compared to the parental peptides. Consistent with our previous observations, most cells co-produced TNF$\alpha$ and IFN- $\gamma$, whereas only a fraction of the IFN- $\gamma$-positive cells produced IL-2. However, importantly, after stimulation with the analogue peptide both the fraction of IL-2-producing cells and the amount of IL-2 produced on a per cell basis were highly increased.

Full CTL activation is associated with cell division and induction of cytolytic effector function. We have previously shown that these functions have the highest requirements, i.e. that more strongly agonistic peptides and/or higher peptide concentrations are required for the induction of expansion and cytolytic effector function as compared to target cell lysis or induction of cytokine synthesis (31). For example, the low potential of parental peptides to induce cytokine production of specific CTL may suggest that these peptides are inefficient activators of cytolytic effector function. To test this, we stimulated peripheral blood lymphocytes from patient LAU 203 with autologous antigen-presenting cells loaded with parental or analogue peptides. After 3 weeks, the cultures stimulated with the parental peptides contained 1.7 and 3.5\% A2/ Melan-A peptide tetramer-positive cells (Fig. 5A), whereas the analogue peptides induced a much larger expansion of antigen-specific CD8 ${ }^{+} \mathrm{T}$ cells (11.2-37.0\% A2/Melan-A peptide tetramer-positive cells). Likewise, the cytotoxicity displayed by cells activated by the parental peptides was weak, whereas activation by the analogue peptides resulted in strong cytotoxicity against target cells pulsed with the parental decapeptide (Fig. 5B) as well as against autologous tumor cells (not shown). This strong cytotoxicity was caused by (i) a high frequency of antigen-specific CTL in the cultures and (ii) a strong activation of these cells, as can be deduced from the data above. In conclusion, the same hierarchy of peptide immunogenicity established above was also found at the level of CTL expansion and induction of cytotoxic effector function.

\section{Discussion}

The analysis of interactions between the TCR and the peptide $\mathrm{MHC}$ class I complex has been until now performed with $\mathrm{T}$ 

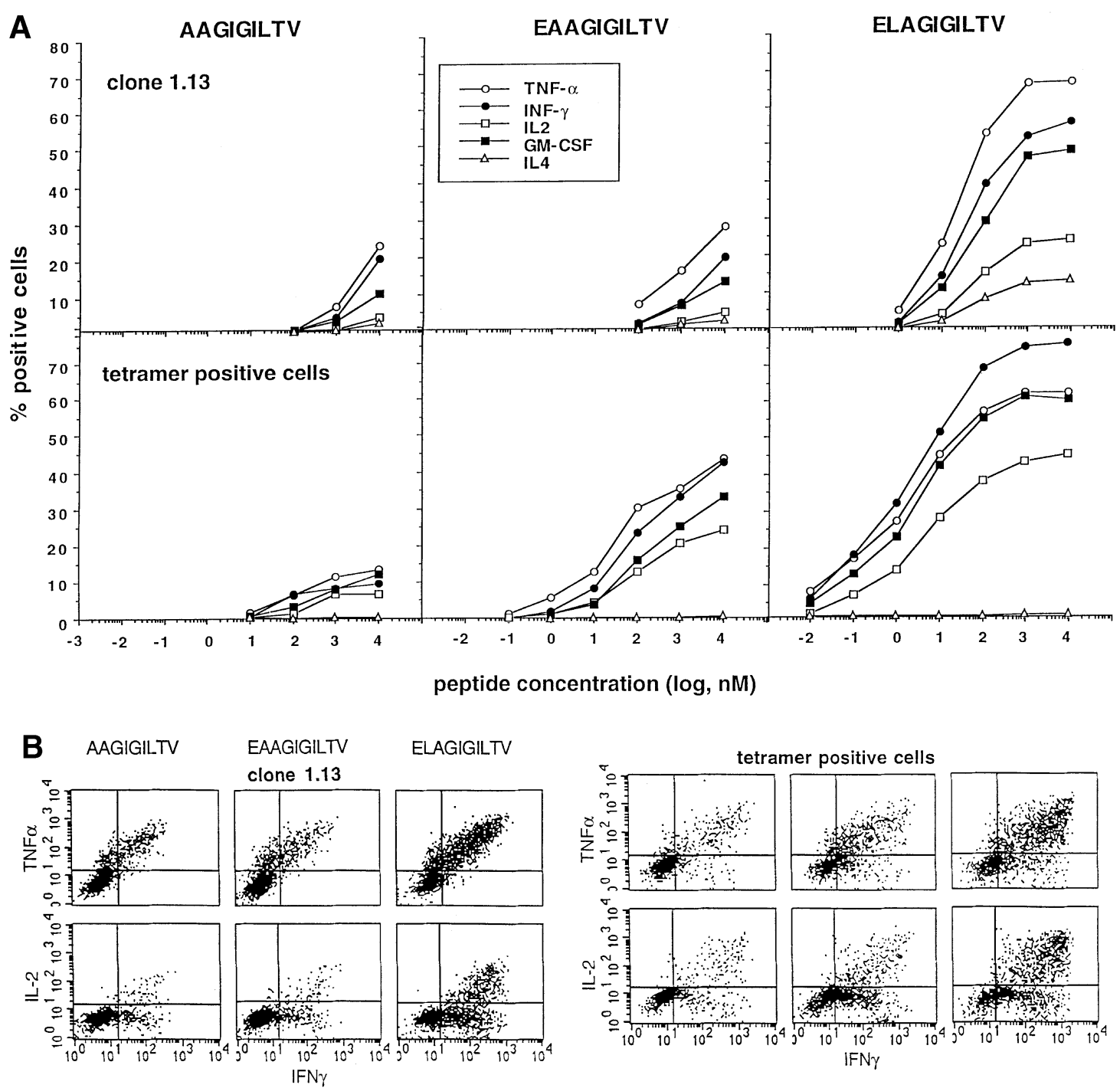

Fig. 4. Induction of cytokine production by Melan-A parental and analogue peptides. Cytokines were measured in the Melan-A-specific $T$ cell clone 1.13 and the tetramer-positive population after peptide stimulation, by intracellular fluorescence labeling and flow cytometry. (A) While the parental nonapeptide (left panel) and decapeptide (middle panel) induced cytokines in only small fractions of the cells, the peptide analogue (right panel) induced high-level cytokine production in higher percentages of cells. However, no IL-4 production was detectable for the tetramer-positive population. (B) Simultaneous staining for two cytokines, TNF- $\alpha$ and IFN- $\gamma$ (upper row) or IL-2 and IFN- $\gamma$ (lower row), showed that the majority of cells co-produced TNF- $\alpha$ and IFN- $\gamma$, whereas only a fraction of IFN- $\gamma$ producing cells co-synthesized IL-2.

cell clones or lymphocytes from TCR transgenic mice $(21,22)$. However, because such $T$ cells are highly selected and are thus not necessarily representative of the polyclonal $T$ cell population specific for a given antigen, the results of such studies are difficult to interpret. For example, it has been shown that various $T$ cell clones specific for a given peptide$\mathrm{MHC}$ class I complex may strongly differ in their effector functions and may also cause drastically different effects in vivo upon adoptive transfer (33). Diversity of TCR fine specificity may be a major cause for these differences. Given the large number of $\mathrm{T}$ cell receptors involved in the CTL response against a given peptide- $\mathrm{MHC}$ class I complex, the heterogeneity of the effector functions observed is not surprising.
In order to assess the CTL response more comprehensively, it is necessary to study $T$ cell populations which are representative of the majority of antigen-specific CTL. The newly developed tetramers consisting of four HLA-A*0201 molecules, four Melan-A peptides and a fluorescent dye allow us to visualize and isolate polyclonal $T$ cell populations by flow cytometry (9). In this study we took advantage of this new technology to assess the activation requirements of MelanA-specific polyclonal T cells isolated from a TILN. Our results show that despite the high degree of immunodominance of the Melan-A antigenic peptide, the parental nonapeptides and decapeptides are weak agonists since they activate antigen-specific cells only suboptimally. In contrast, the Melan-A peptide analogues that we have recently identified 
A

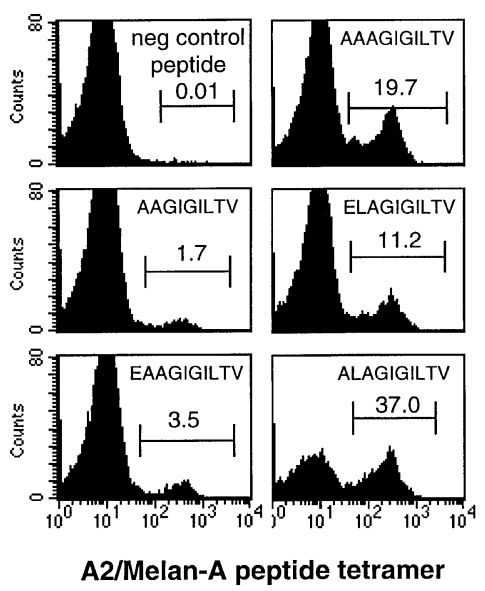

B

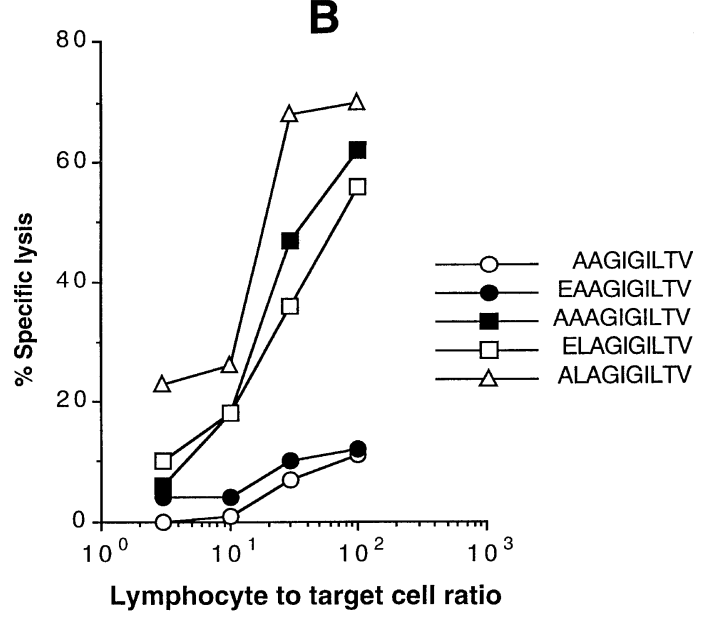

Fig. 5. Induction of Melan-A-specific T cell expansion and cytotoxic effector function by Melan-A parental and analogue peptides. Peripheral blood lymphocytes of patient LAU 203 were stimulated with parental or analogue Melan-A peptides and cultured for 3 weeks as described in Methods. (A) Flow cytometry revealed that the percentages of A2/Melan-A tetramer-positive cells varied strongly depending on the peptide used for stimulation. The parental peptides induced a moderate level of specific cell expansion, whereas high percentages of A2/Melan-A tetramer-positive cells were obtained by stimulation with peptide analogues. (B) Cultures were tested for cytotoxicity in a ${ }^{51} \mathrm{Cr}$-release assay. The two cultures stimulated with the parental peptides (circles) showed weak specific cytolysis. In contrast, strong cytolytic activity was observed in the peptide analogue stimulated cultures (squares and triangle). The values represent the difference between percent specific lysis of peptide loaded minus control targets, the latter values were always $<15 \%$.

behave as full agonists in that they induced a full $\mathrm{T}$ cell activation leading to strong tumor antigen-specific CTL response.

By using Melan-A-specific CTL clones we have previously reported diversity in the efficiency of recognition of nonapeptides and decapeptides both in terms of avidity and fine specificity. However, for the majority of clones analyzed halfmaximal lysis was achieved only in the presence of relatively high concentrations of parental nonapeptide and/or decapeptide (24). Similarly, for the A2/Melan-A peptide tetramerpositive population, half-maximal lysis of target cells required high peptide concentrations (in the micro- to nanomolar range). These findings indicate that the avidity of the majority of Melan-A-specific T cells for the parental Melan-A peptides is relatively low as compared to CTL specific for viral antigens (21). The low avidity toward the parental Melan-A peptides may be explained by the phenomenon of self tolerance. Presumably, high-avidity $T$ cells specific for self antigens are deleted from the repertoire by negative thymic selection $(34,35)$ and only low-avidity autoreactive $T$ cells may escape deletion.

We have previously shown that the Melan-A parental nonapeptides and decapeptides bind to the HLA-A*0201 allele with low affinity and form unstable HLA-A*0201 peptide complexes (23). Indeed, both Melan-A antigenic peptides lack the major anchor residue at position $2(36,37)$. Moreover, peptide Melan-A 26-35 has a glutamic acid at position 1 whose presence was reported to have negative effects on peptide binding to HLA-A*0201 (38). Thus, Melan-A peptide analogues containing a major anchor residue at position 2 (leucine) and/or alanine instead of glutamic acid at position 1 of the decapeptide were designed to improve the binding to HLA-A*0201 and the stability of HLA-A*0201 peptide complexes $(23,24)$. In addition, these analogues were recog- nized with highly improved efficiency by a large fraction of Melan-A-specific T cell clones. The present report shows that the same effect is detectable in polyclonal monospecific $T$ cell populations

The improved binding of the Melan-A analogues does not completely explain their improved recognition by $T$ cells since (i) for some $T$ cell clones the increased recognition ( $>10,000-$ fold) largely exceeded the effect of the amino acid substitution on peptide binding, (ii) the extent of improved recognition was clone dependent and (iii) substitution of leucine for alanine at position 2 of the Melan-A 27-35 nonapeptide strongly improved peptide binding to HLA-A*0201 but resulted in a dramatic decrease in $T$ cell recognition by the majority of specific CTL even at high concentrations $(23,24)$.

Altogether, these data suggest that there may be two A2/ Melan-A peptide conformations. For simplicity we may call them nonapeptide and decapeptide conformations. Introduction of Leu at position 2 of the nonapeptide fixes the nonapeptide conformation. The same substitution at position 1 of the nonapeptide (or at position 2 of the decapeptide) strongly favors the decapeptide conformation. It appears that while the nonapeptide conformation is deleterious for interaction with specific TCR, stabilization of the decapeptide mode of binding results in full activation of the vast majority of specificities present in the Melan-A T cell repertoire.

Thus, the present peptide analogues constitute a unique category of full agonists in which substitution of amino acids either suboptimal or deleterious for $\mathrm{MHC}$ binding leads to stabilization of a ligand conformation which is greatly favored by the large majority of specific TCR. This effect is different from the one obtained using altered peptide ligands carrying substitutions at key TCR contact residues (superagonists) for which the heteroclitic effect on $T$ cell recognition is by definition clone specific. In addition, stimulation by superagonist ligands 
modifies the profile of cytokine produced by $\mathrm{T}$ cells, a phenomenon which has been associated with the capacity of superagonist ligands to alter the course of autoimmune disease $(20,39)$. However, the peptide analogues analyzed in the present study did significantly influence cytokine production but only quantitatively and not qualitatively (Fig. 4A and B). Thus, the new category of full agonist ligands may be more relevant than superagonists for the design of cancer vaccines. Indeed, in vitro stimulation of peripheral blood lymphocytes from melanoma patients with these peptide analogues has demonstrated their high potential at inducing rapid and massive effector CTL maturation. Furthermore, immunization of $\mathrm{A} 2 / \mathrm{Kb}$ transgenic mice with peptide analogues has also shown that they are considerable stronger immunogens than the parental Melan-A peptides (25).

In conclusion, our data suggest that, in addition to the active immune evasion mechanisms developed by tumor cells, the intrinsic low capability of tumor-associated antigens to fully activate tumor-specific $T$ cells may account for the lack of effectiveness of immune responses to tumors. Thus immunization with full agonist analogues may be essential to elicit strong CTL activity against tumor cells in vivo.

\section{Acknowledgements}

This work was partly supported by the Federal Office for Education and Science (OFES), Switzerland, and the European Community (Contract BMH4-CT95-1627). We acknowledge the help in flow cytometry from Pierre Zaech, the excellent technical assistance of Nicole Montandon and the secretarial help of Martine van Overloop.

\section{Abbreviations}

CTL cytotoxic T lymphocyte

PBMC peripheral blood mononuclear cells

TIL tumor-infiltrating lymphocyte

TNF tumor necrosis factor

TILN tumor-infiltrated lymph nodes

\section{References}

1 Robbins, P. F. and Kawakami, Y. 1996. Human tumor antigens recognized by T cells. Curr. Opin. Immunol. 8:628.

2 Boon, T., Coulie, P. and Van den Eynde, B. 1997. Tumor antigens recognized by T cells. Immunol. Today 18:267.

3 Kawakami, Y., Eliyahu, S., Sakaguchi, K., Robbins, P. F., Rivoltini, L., Yannelli, J. R., Appella, E. and Rosenberg, S. A. 1994 Identification of the immunodominant peptides of the MART-1 human melanoma antigen recognized by the majority of HLA-A2restricted tumor infiltrating lymphocytes. J. Exp. Med. 180:347.

4 Kawakami, Y., Eliyahu, S., Delgado, C. H., Robbins, P. F., Rivoltini, L., Topalian, S. L., Miki, T. and Rosenberg, S. A. 1994. Cloning of the gene coding for a shared human melanoma antigen recognized by autologous T cells infiltrating into tumor. Proc. Natl Acad. Sci. USA 91:3515.

5 Coulie, P. G., Brichard, V., Van Pel, A., Wölfel, T., Schneider, J., Traversari, C., Mattei, S., De Plaen, E., Lurquin, C., Szikora, J. P., Renauld, J. C. and Boon, T. 1994. A new gene coding for a differentiation antigen recognized by autologous cytolytic $T$ lymphocytes on HLA-A2 melanomas. J. Exp. Med. 180:35.

6 Spagnoli, G. C., Schaefer, C., Willimann, T. E., Kocher, T., Amoroso, A., Juretic, A., Zuber, M., Luscher, U., Harder, F. and Heberer, M. 1995. Peptide-specific CTL in tumor-infiltrating lymphocytes from metastatic melanomas expressing MART-1/ Melan-A, gp100 and tyrosinase genes: a study in an unselected group of HLA-A2.1 positive patients. Int. J. Cancer 64:309.
7 Rivoltini, L., Kawakami, Y., Sakaguchi, K., Southwood, S., Sette, A., Robbins, P. F., Marincola, F. M., Salgaller, M. L., Yannelli, J. R., Appella, E. and Rosenberg, S. A. 1995. Induction of tumorreactive CTL from peripheral blood and tumor-infiltrating lymphocytes of melanoma patients by in vitro stimulation with an immunodominant peptide of the human melanoma antigen MART1. J. Immunol. 154:2257.

8 D'Souza, S., Rimoldi, D., Liénard, D., Lejeune, F., Cerottini, J.C. and Romero, P. 1998. Circulating Melan-A/MART-1 specific cytolytic T lymphocyte precursors in $\mathrm{HLA}-\mathrm{A} 2^{+}$melanoma patients have a memory phenotype. Int. J. Cancer 78:699.

9 Romero, P., Dunbar, P. R., Valmori, D., Pittet, M., Ogg. G. S. Rimoldi, D., Chen, J. L., Liénard, D., Cerottini, J.-C. and Cerundolo, V. 1998. Ex vivo staining of metastatic lymph nodes by class major histocompatibility complex tetramers reveals high numbers of antigen-experienced tumor-specific cytotoxic T Iymphocytes. J. Exp. Med. 188:1641.

10 Cohen, E. P. and Kim, T. S. 1994. Neoplastic cells that express low levels of MHC class I determinants escape host immunity. Semin. Cancer Biol. 5:419.

11 Cromme, F. V., Airey, J., Heemels, M.-T., Ploegh, H. L., Keating, P. J., Stern, P. L., Meijer, C. J. L. M. and Walboomers, J. M. M. 1994. Loss of transporter protein, encoded by the TAP-1 gene is highly correlated with loss of HLA expression in cervical carcinomas. J. Exp. Med. 179:335.

12 Ye, X., McCarrick, J., Jewett, L. and Knowles, B. B. 1994. Timely immunization subverts the development of peripheral nonresponsiveness and suppresses tumor development in simian virus 40 tumor antigen-transgenic mice. Proc. Natl Acad. Sci. USA 91:3916.

13 Garrido, F., Ruiz-Cabello, F., Cabrera, T., Perez-Villar, J. J., LopezBotet, M., Duggan-Keen, M. and Stern, P. L. 1997. Implications for immunosurveillance of altered HLA class I phenotypes in human tumours. Immunol. Today 18:89.

14 Onrust, S. V., Hartl, P. M., Rosen, S. D. and Hanahan, D. 1996. Modulation of L-selectin ligand expression during an immune response accompanying tumorigenesis in transgenic mice. J. Clin. Invest. 97:54.

15 Guilloux, Y., Viret, C., Gervois, N., Le Dréan, E., Pandolfino, M. C., Diez, E. and Jotereau, F. 1994. Defective lymphokine production by most $\mathrm{CD}^{+}$and $\mathrm{CD} 4^{+}$tumor-specific T cell clones derived from human melanoma-infiltrating lymphocytes in response to autologous tumor cells in vitro. Eur. J. Immunol. 24:1966.

16 Gervois, N., Guilloux, Y., Diez, E. and Jotereau, F. 1996. Suboptimal activation of melanoma infiltrating lymphocytes (TIL) due to low avidity of TCR/MHC-tumor peptide interactions. J. Exp. Med. 183:2403.

17 Kersh, G. J. and Allen, P. M. 1996. Essential flexibility in the Tcell recognition of antigen. Nature 380:495.

18 Dressel, A., Chin, J. L., Sette, A., Gausling, R., Hollsberg, P. and Hafler, D. A. 1997. Autoantigen recognition by human CD8 T cell clones: enhanced agonist response induced by altered peptide ligands. J. Immunol. 159:4943.

19 Vergelli, M., Hemmer, B., Kalbus, M., Vogt, A. B., Ling, N., Conlon, P., Coligan, J. E., McFarland, H. and Martin, R. 1997. Modifications of peptide ligands enhancing $T$ cell responsiveness imply large numbers of stimulatory ligands for autoreactive $T$ cells. J. Immunol. 158:3746.

20 Nicholson, L. B., Waldner, H., Carrizosa, A. M., Sette, A., Collins, M. and Kuchroo, V. K. 1998. Heteroclitic proliferative responses and changes in cytokine profile induced by altered peptides: implications for autoimmunity. Proc. Natl Acad. Sci. USA 95:264.

21 Valitutti, S., Muller, S., Dessing, M. and Lanzavecchia, A. 1996. Different responses are elicited in cytotoxic $T$ lymphocytes by different levels of $\mathrm{T}$ cell receptor occupancy. J. Exp. Med. 183:1917.

22 Bachmann, M. F., Oxenius, A., Speiser, D. E., Mariathasan, S., Hengartner, H., Zinkernagel, R. M. and Ohashi, P. S. 1997. Peptide induced TCR-down regulation on naive T cells predicts agonist/ partial agonist properties and strictly correlates with $\mathrm{T}$ cell activation. Eur. J. Immunol. 27:2195.

23 Valmori, D., Fonteneau, J. F., Marañón Lizana, C., Gervois, N., 
Liénard, D., Rimoldi, D., Jongeneel, C. V., Jotereau, F., Cerottini, J.-C. and Romero, P. 1998. Enhanced generation of specific tumor-reactive CTL in vitro by selected Melan-A/MART-1 immunodominant peptide analogs. J. Immunol. 160:1750.

24 Valmori, D., Gervois, N., Rimoldi, D., Fonteneau, J. F., Bonelo, A., Liénard, D., Rivoltini, L., Jotereau, F., Cerottini, J.-C. and Romero, P. 1998. Diversity of the fine specificity displayed by HLA-A*0201restricted CTL specific for the immunodominant Melan-A/MART1 antigenic peptide. J. Immunol. 161:6956.

25 Men, Y., Miconnet, I., Valmori, D., Rimoldi, D., Cerottini, J.-C. and Romero, P. 1999. Assessment of immunogenicity of human MelanA peptide analogues in $\mathrm{HLA}-\mathrm{A}^{*} 0201 / \mathrm{Kb}$ transgenic mice. $\mathrm{J}$ Immunol. 162:3566.

26 Salter, R. D., Howell, D. N. and Cresswell, P. 1985. Genes regulating HLA class I antigen expression in T-B lymphoblast hybrids. Immunogenetics 21:235.

27 Ogg, G. S., Jin, X., Bonhoeffer, S., Dunbar, P. R., Nowak, M. A., Monard, S., Segal, J. P., Cao, Y., Rowland-Jones, S. L., Cerundolo, V., Hurley, A., Markowicz, M., Ho, D. D., Nixon, D. F. and McMichael, A. J. 1998. Quantitation of HIV-1-specific cytotoxic T lymphocytes and plasma load of viral RNA. Science 279:2103.

28 Valitutti, S., Dessing, M., Aktories, K., Gallati, H. and Lanzavecchia A. 1995. Sustained signaling leading to $T$ cell activation results from prolonged $T$ cell receptor occupancy. Role of $T$ cell actin cytoskeleton. J. Exp. Med. 181:577.

29 Jung, T., Schauer, U., Heusser, C., Neumann, C. and Rieger, C 1993. Detection of intracellular cytokines by flow cytometry. J. Immunol. Methods 159:197.

30 Romero, P., Gervois, N., Schneider, J., Escobar, P., Valmori, D., Pannetier, C., Steinle, A., Wölfel, T., Liénard, D., Brichard, V., Van Pel, A., Jotereau, F. and Cerottini, J.-C. 1997. Cytolytic
T lymphocyte recognition of the immunodominant HLA-A*0201 restricted Melan-A/MART-1 antigenic peptide in melanoma. J. Immunol. 159:2366.

31 Bachmann, M. F., Sebzda, E., Kündig, T. M., Shahinian, A., Speiser, D. E. Mak, T. W. and Ohashi, P. S. 1996. T cell responses are governed by avidity and co-stimulatory thresholds. Eur. J. Immunol. 26:2017.

32 Fonteneau, J. F., Le Dréan, E., Le Guiner, S., Gervois, N., Diez, E. and Jotereau, F. 1997. Heterogeneity of biologic responses of melanoma-specific CTL. J. Immunol. 159:2831.

33 Speiser, D. E., Kyburz, D., Stübi, U., Hengartner, H. and Zinkernagel, R. M. 1992. Discrepancy between in vitro measurable and in vivo virus neutralizing cytotoxic T cell reactivities: Iow T cell receptor specificity and avidity sufficient for in vitro proliferation or cytotoxicity to peptide coated target cells but not for in vivo protection. J. Immunol. 149:972.

34 Nossal, G. J. V. 1994. Negative selection of lymphocytes. Cell $76: 229$

35 von Boehmer, H. 1994. Positive selection of lymphocytes. Cell $76: 219$

36 Falk, K., Rötzschke, O., Stevanovic, S., Jung, G. and Rammensee, H. G. 1991. Allele-specific motifs revealed by sequencing of selfpeptides eluted from MHC molecules. Nature 351:290.

37 Rammensee, H. G., Friede, T. and Stevanovic, S. 1995. MHC ligands and peptide motifs: first listing. Immunogenetics 41:178.

38 Ruppert, J., Sidney, J., Celis, E., Kubo, R. T., Grey, H. M. and Sette, A. 1993. Prominent role of secondary anchor residues in peptide binding to HLA-A2.1 molecules. Cell 74:929.

39 Nicholson, L. B., Greer, J. M., Sobel, R. A., Lees, M. B. and Kuchroo, V. K. 1995. An altered peptide ligand mediates immune deviation and prevents autoimmune encephalomyelitis. Immunity 3:397. 